\title{
Kernkondensationen von Thiophenoläthern I \\ von
}

\section{A. Bistrzycki und Franz Kuba.}

(16. X. 21.)

Die Phenole und ihre Alkyläther sind bekanntlich bereits zu sehr zahlreichen Kernkondensationen mit gutem Erfolge verwendet worden. Für die Thiophenole und ihre Äther gilt das Gleiche nicht. Wir haben in der Literatur keine Angaben über eine Kernkondensation eines Thiophenols auffinden können und nur zwei Mitteilungen über solche Reaktionen von Thiophenoläthern:

Schon vor langer Zeit liessen Auwers und Beger ${ }^{1}$ ) Acylchloride auf Thiophenoläther bei Gegenwart von Aluminiumchlorid einwirken und erhielten Alkylmercapto-ketone, z. B.

$$
\mathrm{C}_{6} \mathrm{H}_{5} \cdot \mathrm{CO} \cdot \mathrm{C}_{6} \mathrm{H}_{4} \cdot \mathrm{S} \cdot \mathrm{C}_{2} \mathrm{H}_{5} .
$$

Zwanzig Jahre später kondensierten Zincke und Ebel ${ }^{2}$ ) Phthalsäure-anhydrid mit m-Oxy-thioanisol zu Bis-(methylmercapto)-3,6fluoran ${ }^{3}$ ).

Was die Thiophenole selbst anbetrifft, so dürften sie sich aus zwei Gründen zu Kernkondensationen wenig eignen. Einmal gehen sie ausserordentlich leicht (z. B. oft schon durch kalte, konz. Schwefelsäure) in Disulfide über, die, soweit bekannt, nur geringe oder gar keine Neigung zu Paarungsreaktionen besitzen. In andern Fällen reagiert aber das Wasserstoffatom der Mercaptogruppe mit der zweiten Komponente des Reaktionsgemisches, indem wiederum die beabsichtigte Kernkondensation ausbleibt ${ }^{4}$ ). Warum wurden aber die Thiophenoläther in dieser IIinsicht bisher vernachlässigt, bei denen die eben genannten Hinderungsgründe ausgeschlossen sind? Reagieren sie wider Erwarten nicht gleich den Phenoläthern, oder liefern sie schwer fassbare Produkte?

1) B. 27, 1733 (1894).

2) B. 47, 925 (1914).

3) In der Originalahhandlung S. 932 als Disulfmethylfluoresceïn bezcichnet.

4) Vgl. hierüber J. Schmid, Diss. S. 11 ff. Freiburg, Schweiz, 1921. 
Um diese Fragen zu entscheiden, haben wir begonnen, Kontensationsversuche mit Thiophenoläthern anzustellen. Wenn wir zu solchen Versuchen zuerst die Benzilsäure herangezogen haben, so geschah dies nicht allein wegen der im hiesigen Laboratorium oft erprobten Leichtigkeit, mit der ihr tertiär-alkoholisches Hydroxyl reagiert, sondern auch vor allem deshalb, weil man hoffen durfte. auf diesem Wege zu dem interessanten 1 -'Thiofuchson, $\left(\mathrm{C}_{6} \mathrm{H}_{5}\right)_{2} \mathrm{C}_{2} \mathrm{C}_{6} \mathrm{I}_{4}: \mathrm{S}$, zu gelangen, in derselben oder in ähnlicher Weise, wie Bistrzyclii und Herbst ${ }^{1}$ ) auts Benzilsäure und Phenol das p-Fuchson selbst crhalten hahen (.. u.).

$$
\begin{aligned}
& \text { p-Methylmercapto-triphenylessigsä̈re. } \\
& \qquad\left(\mathrm{C}_{6} \mathrm{H}_{5}\right)_{2} \mathrm{C} \cdot \mathrm{C}_{6} \mathrm{H}_{4} \cdot \mathrm{S} \cdot\left(\mathrm{CH}_{3}\right. \\
& \mathrm{COOH}
\end{aligned}
$$

\%u einer unter Rückfluss gelinde siedenden Lösung ron $11,4 \mathrm{gr}$ Benzilsäure (1 Mol.) und 7,5 gr Phenylmethyl-thioüther ('Thio-anisol ${ }^{2}$ ) (11/4. Mol.) in $150 \mathrm{~cm}^{3}$ Benzol fügt man in Auteilen $16 \mathrm{gr}\left(=7,6 \mathrm{~cm}^{3}\right)$ wasserfreies Zinntetrachlorid (1 $1 / 4$ Mol.) hinzu und hält diese Mischung hierauf noch 35 bis 40 Minuten im Sieden. The erst gelbliche linissigkeit färbt sich dabei schliesslich tief rotbraun. Man lässt sie erkalten und schüttelt sie zunächst mit ganz verdünnter Salzsäure durch, um die Ilydratationsprodukte des Zinntetrachlorides zu entfernen. Hierauf wird die abgehobene Benzolschicht mit Wasser und endlich mehrmals: mit warmer $\left(50^{0}\right) 5$-proz. Sodalösung durchgeschüttelt. Letztere wird filtriert und mit verdünnter Salzsäure übersattigt, wohei die gebildete Carbonsäure als schwach gelbliche, amorphe Masse ausfällt. Sie wird ahgesaugt, mit warmem Wasser gut ausgewaschen und ans Eisessig Wasser oder Methylalkohol + Wasser umkrystallisiert. IDie Ausbeute an Rohprodukt beträgt gewöhnlich $15,7 \mathrm{gr}$, ist also nahezu quantitativ. Doch kommt es auch vor, dass die Sodalösung der Benzolschicht die entstandene Carbonsäure nicht völlig entzieht. I Iinterlassen einige $\mathrm{cm}^{3}$ bein Eindampten einen nennenswerten Rürksitand, so destilliert man das gesamte Benzol auf dem Wasserbade al, löst die hinterbleibenden gelben Krusten in stark verdünnter, warmer Kalilauge, filtriert und fällt aus dem Filtrat den Rest der Carbonsäiture mit Salzsäure aus.

1) B. 36, 2335 (1903).

2) Dieses wurde dargestellt nach der schönen Methode von Tahoury (A. ('h. [8] 15, 23 [1908]; vergl. C. 1908, II, 1350), nämlich durch lïinwirkung von Mcthyljodid auf Phenylthiomagnesiumbromid (aus Phenylmagnesiumbromid und sichwefel). Ausbeute $60 \%$ der theoretischen. 
Die zwei- bis dreimal umkrystallisierte Säure beginnt bei $204^{\circ} \mathrm{zu}$ erweichen, und schmilzt bei $208-210^{\circ}$. Sie bildet farblose, lanzettförmige Nadeln (aus Methyl- oder Äthylalkohol) oder mikroskopische. flache Prismen (aus Aceton). In der Siedehitze ist sie leicht löslich in Aceton, ziemlich leicht in Methyl- oder Äthylalkohol, mässig in Benzol.

$$
\begin{array}{lllll}
\mathrm{C}_{21} \mathrm{H}_{18} \mathrm{O}_{2} \mathrm{~S} & \text { Ber. C 75,44 } & \text { H } 5,43 & \text { S 9,58\% } \\
& \text { Gef. , 75,21 } & , 5,62 \quad, 9,74 \%
\end{array}
$$

Die Analysenzahlen entsprechen der erwarteten Methylmercaptotriphenylessigsäure. Dass der Benzilsäurerest in die Parastellung zur Gruppe $-\mathrm{SCH}_{3}$ eingetreten ist, wurde zwar bisher nicht experimentell bewiesen, darf aber mit ziemlicher Sicherheit angenommen werden (Vgl. weiter unten beim Methylmercapto-triphenylcarbinol).

Das Silbersalz fällt aus einer Lösung der Säure in Ammoniak, dessen C'berschuss durch Einleiten von Luft vertrieben worden ist, auf Zusatz, von Silbernitrat als weisser. käsiger Niederschlag.

$$
\begin{aligned}
\mathrm{C}_{21} \mathrm{H}_{12} \mathrm{O}_{2} \mathrm{SAg} & \text { Ber. Ag 24,47\% } \\
& \text { Gef. , 24,21\% }
\end{aligned}
$$

Versuche zur Entmethylierung der Methylmercapto-triphenylessigsäure führten nicht zum Ziel. Mit Jodwasserstoffsäure (spez. Gew. 1,7) vier Stunden im geschlossenen Rohr auf $200^{\circ}$ erhitzt, blieb sie noghh zum Teil unverändert. Bei achtstündigem Erhitzen auf $220^{\circ}$ ergab sie eine ölige, fast schwarze Masse, die sich nicht näher charakterisieren liess.

Mit Aluminiumchlorid ${ }^{2}$ ) und Schwefelkohlenstoff oder Benzol ${ }^{3}$ ) gekocht, blieb die Säure unverändert. Wurde sie mit fein gepulvertem Aluminiumehlorid ohne Lösungsmittel erhitzt, so konnte man zwar bisweilen von $120^{\circ}$ an eine Abspaltung von Methylchlorid bemerken, aber auch in diesem Falle liess sich (las hinterbleibende Produkt nicht fassen ${ }^{4}$ ).

$$
\begin{aligned}
& \text { p-Methylmercapto-triphenylessigsäure-methylester. } \\
& \qquad\left(\mathrm{C}_{6} \mathrm{H}_{5}\right)_{2} \mathrm{C} \cdot \mathrm{C}_{6} \mathrm{H}_{4} \cdot \mathrm{S} \cdot \mathrm{CH}_{3} \\
& \mathrm{COO} \cdot \mathrm{CH}_{3}
\end{aligned}
$$

In üblicher Weise mit Dimethylsulfat in alkalischer Lösung methyliert, liefert die eben beschriebene Säure einen Methylester, der aus Eisessig + Wasser oder Methyl-

1) Die ausführlichen Analysenprotokolle, sowie sonstige Einzelheiten finden sich in der Dissertation von $F$. Kubn, Freiburg, Schweiz, 1915.

2) Hartmann und Gattermann, B. 25, 353 (1892).

3) O. N. Witt, B. 47, 2216 (1914).

4) Vergl. die analogen Erfahrungen von Auwers und Arndt über die Schwierigkeit der Entmethylierung gewisser Thiophenoläther, B. 42, 533 ff. (1909). 
alkohol + Wasser in farblosen, zu Drusen vereinigten Prismen vom Simp. 141-142,50 krystallisiert. In der Hitze ziemlich leicht löslich in Benzol, schwerer in Methylalkohol. noch schwerer in Jigroīn.

$$
\begin{aligned}
& \mathrm{C}_{22} \mathrm{H}_{20} \mathrm{O}_{2} \mathrm{~S} \quad \text { Ber. C 75,86 H .j,54\% } \\
& \text { Gef. ," 75,68 , ,5,64 } \\
& \text { p-Methylmercapto-triphenylcarbinol. } \\
& \begin{array}{c}
\left(\mathrm{C}_{6} \mathrm{H}_{5}\right)_{2} \mathrm{C} \cdot \mathrm{C}_{6} \mathrm{H}_{4} \cdot \mathrm{S} \cdot \mathrm{CH}_{3} \\
\mathrm{OH}
\end{array}
\end{aligned}
$$

Übergiesst man die p-Methylmercapto-triphenylessigsäure mit reiner Schwefelsäure (spez. Gew. 1,84), so geht sie schon bei gewöhnlicher 'Temperatur unter Entwicklung von Kohlenoxyd mit tief kirschroter' Farbe in Lösung. Zur quantitativen Bestimmung des abgespaltenen Kohlenoxyds wurde die Versuchsanordnung ron Bistrzychi und $v$.Siemiradzkil) benutzt und die Mischung erwärmt. Die Abspaltung war bei $80^{\circ}$ völlig beendet. Schwefeldioxyd trat dirbei nicht auf.

$$
\begin{array}{ll}
\mathrm{C}_{21} \mathrm{H}_{18} \mathrm{O}_{2} \mathrm{~S}-\mathrm{CO} & \text { Ber. CO 8,38" } \\
& \text { Gef. " } 7,56 \text { und } 7,97^{\prime \prime} 。
\end{array}
$$

Die Entcarbonylierung war also nahezu quantitatir.

Zur präparativen Darstellung des hicrbei entstandenen Carbinols wurden $10 \mathrm{gr}$ der Säure mit $60 \mathrm{~cm}^{3}$ (nicht mehr!) 95-proz. Schwefelsäure sechs bis acht Stunden bei Zimmertemperatur unter zeitweisen Tmschwenken stehen gelassen. Dann wurde die schwefelsaure Lösung in 1,5 bis 2 Liter Wasser gegossen und das amorph ausfallende, gelblich gefärbte Carbinol nach gutem Auswaschen nit Wasser aus 70-80-proz. Essigsäure oder aus Methylalkohol umkrystallisiert. Nan erhält so gelblich gefärbte Prismen in einer Ausbeute von $6,6 \mathrm{gr}=72 \%$ der Theorie. Auch bei mehrmaliger Krystallisation verschwindet ihre Färbung nicht völlig. Sie schmelzen konstant. nach kurz vorhergehendem Erweichen, bei 65 bis $67^{\circ}$, ganz klar erst hei $68^{\circ}$.

$$
\begin{aligned}
& \left(_{20} \mathrm{H}_{18} \text { OS Ber. C 78,38 H 5,92 } \quad \therefore 10,47 \%\right. \\
& \text { Gef. ,78,14 ,6,09 , } 10,30 \%_{\circ}
\end{aligned}
$$

In Benzol, Aceton oder Schwefelkohlen toff löst sich das Carbinol schon in der Kälte leicht, ebenso in siedendem Ligroin. V'on kon\%. Schwefelsäure wird es mit rotvioletter Farlse aufgenommen, die einen Stich ins Bräunliche aufweist. Dieselbe Farbe tritt auf, wenn man die kalte Lösung des Carbinols in Eisessig mit rauchender Salzsäure ver-

1) B. 39, 53 (1906). 
setzt, oder die Lösung in reinem Eisessig erhitzt. Wird letztere Lösung mit Wasser verdünnt, so schlägt die Farbe in ein schwaches Gelb um, und schliesslich scheidet sich das Carbinol unverändert wieder aus.

Während der Niederschrift der vorliegenden Abhandlung, deren Ergebnisse (abgesehen von wenigen Zusätzen) bereits 1915 in der zitierten Dissertation des einen von uns niedergelegt worden sind, erschien eine interessante vorläufige Mitteilung von $K$. Brand und Stallmann ${ }^{1}$ ), welche durch Einwirkung der aus dem Methyläther des o-Brom-thiophenols (nach Grignard) erhaltenen Magnesiumverbindung auf Benzophenon zu dem Orthoisomeren unseres p-Methylmercapto-triphenylcarbinols gelangt sind. Es schmilzt bei $96^{\circ}$ und gibt, in Eisessig gelöst, auf Zusatz von konzentrierter Schwefelsäure eine gelbstichig grüne Färbung, ist also sicher von unserm Carbinol verschieden, dessen Auffassung als Paraverbindung dadurch gestützt wird. Die genannten Forscher erwähnen, dass das Paraisomere (von Dr. Vogt) auf anderm Wege erhalten wurde, über den später berichtet werden soll, und dass es mit Eisessig-Schwefelsäure eine rotviolette Lösung liefert, was mit unserm Befunde übereinstimmt.

\section{p-Methylmercapto-triphenylmethan.}

$$
\left(\mathrm{C}_{6} \mathrm{H}_{5}\right)_{2} \mathrm{CH} \cdot \mathrm{C}_{6} \mathrm{H}_{4} \cdot \mathrm{S} \cdot \mathrm{CH}_{3} \cdot
$$

Reduktion des Carbinols mit Zinkstaub und Eisessig führt bei mehrstündigem Kochen des Gemisches (bis zur Entfärbung) zu dem entsprechenden Methan, das sich beim Eingiessen der Lösung in Wasser als gelbliche, amorphe Masse ausscheidet. Es krystallisiert aus Alkohol oder Eisessig, in denen es in der Hitze leicht löslich ist, in zu Büscheln vereinigten, farblosen Nadeln vom Smp. 68-69 ${ }^{\circ}$. Sie sind in Benzol schon in der Kälte, in Ligroin in der Hitze leicht löslich.

$$
\begin{array}{llll}
\mathrm{C}_{20} \mathrm{H}_{18} \mathrm{~S} & \text { Ber. C 82,75 } & \mathrm{H} 6,21 & \mathrm{~S} 11,03 \% \\
& \text { Gef. „82,69 } & \text {, } 6,57 & \text { " } 10,94 \%
\end{array}
$$

Der reine, mehrfach krystallisierte Methankörper gibt beim Übergiessen mit konz. Schwefelsäure keine Färbung. Erst bei längerem Schütteln mit der Säure oder beim Erwärmen tritt eine intensive Rotfärbung auf, vielleicht infolge einer teilweisen Oxydation zum Carbinol.

1) B. 54, 1578 (Juliheft 1921). 


$$
\begin{gathered}
--974- \\
p \text {-Methylmercapto-triphenyl-chlormethan. } \\
\left(\mathrm{C}_{6} \mathrm{H}_{5}\right)_{2} \mathrm{CCl} \cdot \mathrm{C}_{6} \mathrm{H}_{4} \cdot \mathrm{S} \cdot \mathrm{CH}_{3}
\end{gathered}
$$

Das p-Fuchson ist bekanntlich zuerst durch Erhitzen des dem p-Methoxy triphenylcarbinol entsprechenden Chlorides erhalten worden ${ }^{1}$ ):

$$
\left(\mathrm{C}_{6} \mathrm{H}_{5}\right)_{2} \mathrm{CCl} \cdot \mathrm{C}_{6} \mathrm{H}_{4} \cdot \mathrm{OCH}_{3}=\left(\mathrm{C}_{6} \mathrm{H}_{5}\right)_{2} \mathrm{C}: \mathrm{C}_{6} \mathrm{H}_{4}: \mathrm{O}+\mathrm{CH}_{3} \mathrm{Cl}
$$

Um den analogen Versuch mit dem obigen Methylmercaptocarbinol anstellen zu können, wurde dieses zunächst in das zugehörige Chlorid übergeführt, indem in eine Lösung von $5 \mathrm{gr}$ des reinen und gut getrockneten Carbinols in $80 \mathrm{~cm}^{3}$ abs. Äther $1 \frac{1}{2}$ Stunden lang trockener Chlorwasserstoff eingeleitet wurde. Dabei färbte sich die Lösung rot und erwärmte sich, indem der Äther langsam abilestillierte und das Chlorid sich als rhombische, nur schwach gefärbte Täfelchen rom Smp. $88,5-89,5^{\circ}$ abschied.

$$
\begin{array}{llllll}
\mathrm{C}_{20} \mathrm{H}_{17} \mathrm{SCl} & \text { Ber. C 73,96 } & \text { H 5,24 } & \text { S 9,88 } & \text { Cl } 10,92 \% \\
& \text { Gef. ,, } 74,49 & , & \mathbf{5 , 4 3} & , 9,83 & ., 10,73 ; 10,69 \%
\end{array}
$$

Nun wurde das Chlorid in einem Strome von sorgfältigst getrocknetem Kohlendioxyd erhitzt. Dabei trat bei etwa $160^{\circ}$ starke Gasentwicklung ein, die bis $260^{\circ}$ andauerte. Statt der erwarteten blossen Methylchlorid-Abspaltung fand aber eine tiefer gehende Zersetzung statt, was sich aus der Bildung von Salzsäuregas schliessen liess. Der Rückstand, eine braunschwarze, klebrige Masse, konnte nicht charakterisiert werden, da er zwar in wasserfreien Lösungsmitteln, wie Benzol, löslich war, aber nicht zur Krystallisation gebracht werden konnte.

Mehrere Wiederholungen dieses Versuches ergaben leider keine günstigeren Resultate, sodass, wenigstens vorläufig, auf die Gewinnung der Thiofuchsons auf diesem Wege verzichtet werden musste.

$$
\begin{gathered}
\text { p-(Methyl-sulfoxyd)-triphenylessigsäure } \left.{ }^{2}\right) . \\
\qquad\left(\mathrm{C}_{6} \mathrm{H}_{5}\right)_{2} \mathrm{C} \cdot \mathrm{C}_{6} \mathrm{H}_{4} \cdot \mathrm{SO} \cdot \mathrm{CH}_{3} \\
\text { COOH }
\end{gathered}
$$

Die nun unternommenen Versuche, die p-Methylmercapto-triphenylessigsäure zu bromieren, hatten den $Z$ weck, die erwartete bromierte Säure in ein Bromcarbinol und dieses dann in ein Bromthiofuchson zu verwandeln, das leichter fassbar sein konnte als die nicht bromierte Verbindung. Es zeigte sich indessen, dass das Brom in Benzol

1) Bistrzycki und Herbst, B. 36, 2335 (1903); vergl. Graf Rostworowski, Diss. S. 36. Freiburg, Schweiz, 1911.

2) Oder 4-Methansulfinyl-triphenylessigsäure nach der Nomenklatur in Stelzner's Literaturregister $1910-1911$, S. XVI. 
oder Eisessig nicht auf den Kern des Thio-anisolrestes wirkte, sondern a!lem Anscheine nach sich an das Schwefelatom addierte. Das entstehende Bromid wurde nicht näher untersucht; es gibt sein Brom sehr leicht vollstä̀ndig ab, z. B. beim Umkrystallisieren aus verdünntem Methylalkohol.

Nascierendes Brom in wässriger Lösung oxydiert die Methylmercaptosäure zu einem Sulfoxyd: $2 \mathrm{gr}$ der Säure werden in $20 \mathrm{~cm}^{3}$ 10-proz. Kalilauge gelöst und mit einer unter Kühlung bereiteten Lösung von $4 \mathrm{gr}$ Brom in $30 \mathrm{~cm}^{3}$ der gleichen Kalilauge versetzt. Die Mischung wird mit Wasser auf $500 \mathrm{~cm}^{3}$ verdünnt, filtriert und auf $80^{\circ}$ erwärmt. Setzt man nun tropfenweise einen Úberschuss von verdünnter Schwefelsäure hinzu, so fällt ein weisser, krystallinischer Niederschlag aus, der aus Alkohol in farblosen, mikroskopischen Prismen krystallisiert. Sie zersetzen sich bei $130^{\circ}$ und sind bromfrei.

$$
\begin{array}{lll}
\mathrm{C}_{21} \mathrm{H}_{18} \mathrm{O}_{3} \mathrm{~S} & \text { Ber. C } 70,20 & \text { H } 5,02 \% \\
& \text { Gef. , 69,81 } & \text {, } 5,38 \%
\end{array}
$$

Dass in der Tat ein Sulfoxyd vorliegt, ergibt sich daraus, dass es in alkalischer Lösung mit Kaliumpermanganat leicht zu einem Sulfon oxydierbar ist.

Nach vierjährigem Aufbewahren dieses Sulfoxydes war sein Zersetzungspunkt auf $219-220^{\circ}$ gestiegen, aus welchem Grunde, konnten wir bisher nicht ermitteln.

$$
\begin{gathered}
\text { p-Methylsulfon-triphenylessigsäure } \left.{ }^{1}\right) . \\
\qquad \begin{array}{c}
\left(\mathrm{C}_{6} \mathrm{H}_{5}\right)_{2} \mathrm{C} \cdot \mathrm{C}_{6} \mathrm{H}_{4} \cdot \mathrm{SO}_{2} \cdot \mathrm{CH}_{3} \\
\mathrm{COOH}
\end{array}
\end{gathered}
$$

Das eben erwähnte Sulfon lässt sich aus der p-Methylmercapto-triphenylessigsäure direkt leicht erhalten, wenn man eine Lösung von $8 \mathrm{gr}$ derselben in $100 \mathrm{~cm}^{3} \mathrm{n}$. Kalilauge unter andauerndem Erhitzen auf dem Wasserbade ganz allmählich mit einer 3-proz. Lösung von Kaliumpermanganat so lange (1 $1 \frac{1}{2}$ bis 2 Stunden) versetzt, als dieses noch reduziert wird. Der schliesslich vorhandene kleine Überschuss von Permanganat wird durch \%ugabe von etwas Kaliumformiat reduziert und das ausgeschiedene Mangandioxydhydrat in der Hitze abfiltriert. Wird das noch heisse Filtrat mit Essigsäure angesäuert, so fällt das Sulfon in mikroskopischen Prismen aus. Diese sind in der Hitze ziemlich leicht löslich in Aceton, mässig in Äthylalkohol, ziemlich schwer in Methylalkohol, und sehr schwer in Benzol. Die Verbindung krystallisiert aus Methylalkohol in farblosen rechteckigen Tafeln, die eine Molekel Alkohol enthalten. An der Luft getrocknet und im Schmelzpunktsröhrchen erhitzt, zersetzen sie sich unter starker Gasentwicklung bei $140^{\circ}$.

$$
\begin{array}{lll}
\mathrm{C}_{21} \mathrm{H}_{18} \mathrm{O}_{4} \mathrm{~S}+\mathrm{CH}_{3} \cdot \mathrm{OH} & \begin{array}{l}
\text { Ber. C 66,00 } \\
\text { G } 5,68 \%
\end{array} \\
& \text { Gef. "66,10 }, 6,00 \%
\end{array}
$$

Bei längerem Erhitzen auf $120^{\circ}$ wird der Methylalkohol vollständig abgegeben.

$$
\begin{aligned}
\mathrm{CH}_{3} \mathrm{OH} & \text { Ber. } 8,75 \% \\
& \text { Gef. } 9,03 \text { und } 8,90 \%
\end{aligned}
$$

Analyse der alkoholfreien Substanz:

$$
\begin{aligned}
& \mathrm{C}_{21} \mathrm{H}_{18} \mathrm{O}_{4} \mathrm{~S} \quad \text { Ber. C } 68,85 \quad \mathrm{H} 4,92 \% \\
& \text { Gef. , 68,68 , 4,92\% }
\end{aligned}
$$

1) Nach Stelzner: p-Methansulfonyl-triphenylessigsäure. 
Schnell erhitzt, zersetzt sich die alkoholfreie Verbindung bei etwa $160^{\circ}$, langsam erhitzt, erst wesentlich höher. Sie spaltet nämlich dabei allmählich Kohlendioxyd ab. Als eine Probe im Luftstrom auf $225^{\circ}$ (Badtemperatur) erhitzt wurde, war die Abspaltung von Kohlendioxyd rollständig.

$$
\begin{array}{ll}
\mathrm{C}_{21} \mathrm{H}_{18} \mathrm{O}_{1} \mathrm{~S}-\mathrm{CO}_{2} & \text { Ber. } \mathrm{CO}_{2} 12,02^{\circ}{ }^{\circ} \\
& \text { Gef. ", } 12,42^{\circ}{ }^{\circ}
\end{array}
$$

Mit konz. Schwefelsäure übergossen, cribt die Sulfoncarbonsäure unter Orangefärbung und Aufschäumen Kohlenoxyd al. Bei der quantitativen Bestimmung des letzteren (vergl oben) wurde die Mischung gelinde erwärmt.

$$
\begin{aligned}
& \mathrm{C}_{21} \mathrm{H}_{18} \mathrm{O}_{4} \mathrm{~S}-\mathrm{CO} \text { Ber. } \mathrm{CO} 7,68^{\circ} \\
& \text { Gef. "7,46" }
\end{aligned}
$$

Wie man sieht, erschwert die Anwesenheit der Methylsulfongruppe die Entcarbonylierung keineswegs, erleichtert sie sogar in gewissem Grade. Die Triphenylessigsäure selbst' ${ }^{1}$ ) wird nämlich bei Zimmertemperatur nicht (oder nur sehr langsam) entrarbonyliert. I)ie $\mathrm{SO}_{2} \cdot \mathrm{OH}$ Gruppe dagegen pflegt die Entcarbonylierungs-Fähigkeit von Tri-arylessigsäuren bedeutend zu beeinträchtigen, wie Bistrzyclii und $r$. Siemiradzhi ${ }^{2}$ bereits bemerkt haben.

$$
\begin{aligned}
& p \text {-Methylsulfon-triphenylcarbinol } \\
& \text { (p-Methansulfonyl-tripheny-learbinol). } \\
& \left(\mathrm{C}_{6} \mathrm{H}_{5}\right)_{2} \mathrm{C}(\mathrm{OH}) \cdot \mathrm{C}_{6} \mathrm{H}_{4} \cdot \mathrm{so}_{2} \cdot\left(\mathrm{H}_{3}\right.
\end{aligned}
$$

Zur präparativen Darstellung dieses Carbinols werden 5 gr der (methylalkoholhaltigen) Sulfoncarbonsäure mit $50 \mathrm{~cm}^{3}$ konz. Schwefelsäure übergossen und unter häufigem Umschütteln eine Stunde stehen gelassen. Dann erwärnt man die tief orangefarbene Mischung auf $40-50^{\circ}$, wobei die letzten Pesste von Kohlenoxyd entweichen. Beim Eingiessen der erkalteten Lösung in ein bis zwei Liter Wasser schied sich das entstandene Sulfoncarbinol als gelbliche, amorphe Masse aus, die mit warmer, verdünter Sodalösung verrieben und hierauf mit Wasser ausgewaschen wurde. Das Carbinol krystallisiert aus Äthy]- oder Methylalkohol, in denen $\mathrm{s}$ in der Hitze ziemlich schwer löslich ist, in flachen, zugespitzten Prismen. Es ist leicht löelich in heissem Aceton, ziemlich schwer in Benzol. Aus allen diesen Lösungsmitteln wird es hell schwefelgelb gefärbt erhalten. Doch verschwindet diese Färbung, wenn man es mit Zink und Alkali in alkoholischer Lösung einige Minuten kocht. Rerluktion findet dabei nicht statt. Das dreimal krystallisierte (nicht entfärbte) Produkt schmol\% scharf bei $132^{\circ}$.

$$
\begin{aligned}
& \mathrm{C}_{20} \mathrm{H}_{18} \mathrm{O}_{3 \mathrm{~S}} \mathrm{~S} \quad \text { Ber. C 71,00 } \mathrm{H}, 5,33 \% \\
& \text { Gef. , 70,91 , , i, } 46 \%
\end{aligned}
$$

1) Bistrzycki und $G y$, B. 38, 839, Anm. 2, und 1822 (1905).

2) B. 39, 62 (1906). 
Nachdem die Verbindung einige Monate aufbewahrt worden war, wies sie den Smp. $178-179^{\circ}$ auf, der weiterhin konstant blieb. Als die höher schmelzende Form nochmals aus Aceton + Wasser krystallisiert wurde, ging anfangs der Schmelzpunkt wieder auf $132^{\circ}$ zurück. Nach vierjähriger Unterbrechung (durch den Weltkrieg) haben wir diese Versuche wiederholt. Nunmehr erhielten wir sofort Krystalle vom Smp. 178-1790, die beim Umkrystallisieren aus Aceton + Wasser, oder auch aus Benzol (mikroskopische, wetzsteinförmige Blättchen) sich nicht mehr in die bei $132^{\circ}$ schmelzende Form verwandelten. Wurde die im Röhrchen wieder erstarrte Substanz nochmals erhitzt, so schmolz sie abermals bei $178-179^{\circ}$.

$0,1760 \mathrm{gr}$ Subst. gaben $0,4610 \mathrm{gr} \mathrm{CO}_{2}$ und $0,0892 \mathrm{gr} \mathrm{H}_{2} \mathrm{O}$

$$
\begin{array}{lll}
\mathrm{C}_{20} \mathrm{H}_{18} \mathrm{O}_{3} \mathrm{~S} & \begin{array}{l}
\text { Ber. C 71,00 } \\
\text { Gef. , 7 5,33\% }
\end{array} \\
& \text { Ge,79 } & \text {, 5,63\% }
\end{array}
$$

Ob hier Stereoisomerie im Sinne Hinsbergs ${ }^{1}$ ) vorliegt, oder vielleicht Polymorphie, wie bei der cis-Zimtsäure, konnte bisher nicht festgestellt werden.

Die orangegelbe Lösung des Carbinols in konz. Schwefelsäure ist weit weniger intensiv gefärbt als die kirschrote lösung des entsprechenden Methylmercaptocarbinols. Sehr auffällig ist, dass ersteres - im Gegensatz zu letzterem - selbst bei 30-stündigem Kochen mit Zinkstaub und Eisessig, oder mit Zinkstaub und Alkali in alkoholischer Lösung nicht oder kaum reduziert wird. Doch sind Carbinole, die diesen Reduktionsmitteln mehr oder weniger trotzen, bereits bekannt ${ }^{2}$ ).

\section{( $\alpha$-Methylmercapto-naphthyl)-diphenyl-essigsäure}

$$
\begin{aligned}
& \left(\mathrm{C}_{6} \mathrm{H}_{5}\right)_{2} \mathrm{C} \cdot \mathrm{C}_{10} \mathrm{H}_{6} \cdot \stackrel{(\alpha)}{\mathrm{S}} \cdot \mathrm{CH}_{3} \\
& \mathrm{COOH}
\end{aligned}
$$

In der gleichen Weise wie der Methyläther des Thiophenols (s. o.) lässt sich auch der Methyläther des $\alpha$-Thionaphtols ${ }^{3}$ ) mit Benzilsäure in benzolischer Lösung bei Gegenwart von Zinntetrachlorid paaren.

Schüttelt man das erkaltete Reaktionsgemisch mit verdünnter Salzsäure aus, so fällt, schon dabei ein voluminöser Niederschlag aus, oft aber auch erst dann, wenn man versucht, die entstandene Carbonsäure der Benzolschicht mittels verdünnter Soda-

1) J. pr. [2] 93, 302; 94, 176 (1916); vergl. Bistrzycki und Brenken, Helv. 3, 453 and 454 (1920).

2) Vergl. Tschitschibabin, B. 44, 441 (1911).

3) Vergl. Taboury, l. c. 
lösung zu entziehen. In beiden Fällen besteht der Niederschlag aus der freien tertiären Säure, die auf diese Weise fast vollständig abgeschieden wird. Sie wird abgesaugt, mit sehr verdünnter Salzsäure ausgewaschen und aus Aceton + Wasser krystallisiert: Zu Drusen vereinigte, rhombenförmige Blättchen, die bei ctwa $204^{0}$ unter starker Entwicklung von Kohlendioxyd schmelzen, nach Verfärbung und Erweichung. Ausbeute nahezu quantitativ.

$$
\begin{array}{lllll}
\mathrm{C}_{20} \mathrm{H}_{20} \mathrm{O}_{2} \mathrm{~S} & \text { Ber. C 78,13 } & \text { H } & 5,20 & \$ 8,333^{\circ} \\
& \text { Gef. , 77,82 } & , 5,45 & , 8,09{ }^{\circ}
\end{array}
$$

Die Säure ist ziemlich schwer löslich in Areton, schwer in Eisessig und noch schwerer in Alkohol oder Benzol. Auch ihre Alkalisalze sincl selbst in der Hitze verhältnismässig schwer lïslich, loch wird die Säure von heisser, etwa 1\%/2-proz. Sodalösung aufgenommen. Bein Erkalten krystallisiert das Natriumsalz in zu Büscheln veremigten Nadeln aus. Ubergiesst man die Carbonsäure mit konz. Schwefelsäure, so bleiht letztere zunächst ungefärbt. Beim Erwärmen löst sich die Substan\% mit schmutzig olivgrüner Färbung, während, wie oben erwähnt, die Farbe der schwefelsauren Lösung bei dem entsprechenden Prorlukt aus Thioanisol kirschrot ist. Wahrscheinlich beruht dieser Interschied auf einer verschiedenen Konstitution beider Verbindungen, indon der Benzilsäurerest gegenüber der Methylmercaptogrupue' des 'Thionaphtholäthers nicht in die Stellung 4, sondern in 2 eingetreten ist. Dafü spricht die erwähnte Grünfärbung des o-Methylmercapto-triphenylearlinols mit Eisessig-Schwefelsäure.

Der Methylester der vorsteheṇden Säure wurde durch Erwärmen ihrer Lösung in sehr verdünnter (etwa 0,08-proz.) Kalilauge mit Dimethylsulfat erhalten. (In normaler Kalilauge wäre die Säure kaum löslich.) Er krystallisiert aus Aceton + Wasser in farblosen Nadeln vom Smp. 185-186 . In der Hitze leicht löslich in Aceton, sechr wenig in Methylalkohol oder Ligroïn.

$$
\begin{aligned}
& \mathrm{C}_{25} \mathrm{H}_{20} \mathrm{O}_{2} \mathrm{~S} \text { Ber. C 78,13 H } 5.20 \% \\
& \text { Gef. C } 77,86 \quad, \quad \therefore .47 \%
\end{aligned}
$$

Im durch Entcarbonylierung der vorstehenden säure das entsprechende Carbinol zu erhalten, wurde sic mit 96-proz. Schwefelsäurc unter ofterem Imschütteln einige Stunden stchen gelasisen. Es trat \%war Grünfärbung und Gasentwicklung auf, allein das beim Eingiessen ler Lösung in Wasser ausfallende Produkt war rollständig löslich in stark verdünnter Sodalösung und erwies sich zum grössten Teil als unvoränderte Säure.

Als die -Entcarbonylierung mit etwa 94-groz. Sichwefelsäure quantitativ wiederholt wurde, wurden nur $2,90^{\circ}$, statt der herechneten $7,3 \%$ Kohlenoxyd erhalten. Dabei wurde auch das Aufteoten von Schwefeldioxyd ron Anfang an beobachtet. Iun als der Versuch zur 
Gewinnung des Carbinols in der Wärme wiederholt wurde, bildete sich dieses nicht, ehensowenig bei Verwendung von Schwefelsäure-Eisessig ${ }^{1}$ ).

Nun wurde statt der gewöhnlichen konz. Schwefelsäure absolute angewandt. Überraschenderweise färbte sich das Reaktionsgemisch bei Zimmertemperatur nicht mehr grün, sondern rotbraun und beim Erwärmen auf etwa $50^{\circ}$ blauviolett. Erst von etwa $60^{\circ}$ an trat Schwefellioxyd auf. Bei einem entsprechenden präparativen Versuche wurde die Mischung nicht erwärmt, sondern nach dem Aufhören der Kohlenoxydentwicklung, d. h. nach etwa einer Stunde, in Wasser gegossen. Ier dabei ausgeschiedene gallertartige Niederschlag bestand aber wiederum nicht aus dem gewünschten Carbinol, da er in heissem Wasser ziemlich löslich war. Vermutlich lag in ihm eine Sulfosäure vor.

Die quantitative Bestimmung des Kohlenoxyds ergab in diesem Falle wesentlich mehr, nämlich $6 \%$ statt $7,3 \%$. Das entwickelte Gas ging fast völlig bei Zimmertemperatur über, doch wurde die Mischung noch bis auf $80^{\circ}$ erwärmt.

$$
\begin{aligned}
& \text { ( } \alpha \text {-Methylmercapto-naphthyl)-diphenylmethan. } \\
& \left(\mathrm{C}_{6} \mathrm{H}_{5}\right)_{2} \mathrm{C} \mathrm{Ii} \cdot \mathrm{C}_{10} \mathrm{H}_{6} \cdot \mathrm{s} \cdot \mathrm{CH}_{3}
\end{aligned}
$$

Wird die vorliegende Säure in einem Schwefelsäurebad auf $200-225^{\circ}$ bis zum Aufhören der Gasentwicklung erhitzt, so hinterbleibt eine dunkel gefärbte Masse. Diese wurde mit warmer, sehr verdünnter Sodalösung verrieben und durch Kochen ihrer Acetonlösung mit Tierkohle entfärbt. Auf Zusatz von Wasser zu der filtrierten Lösung krystallisierten dann Prismen vom Smp. 157-1580 aus. Die Analyse erwies, dass das erwartete Methanderivat durch Abspaltung von Kohlendioxyd entstanden war.

$$
\begin{array}{rll}
\mathrm{C}_{24} \mathrm{H}_{20} \mathrm{~S} & \text { Ber. C 84,71 } & \text { H 5,88\% } \\
& \text { Gef. , 84,42 } & \text { "6,07\% }
\end{array}
$$

Die Verbindung ist schon in der Kälte leicht löslich in Chloroform, in der Hitze leicht auch in Benzol, mässig in Aceton, sehr weniz in Methylalkohol.

Auch Diphenylthio-äther vermag sich mit Benzilsäure in benzolischer Lösung bei Gegenwart von Zinntetrachlorid zu kondensieren. Doch haben wir das in schlechter Ausbeute erhaltene Produkt bisher

1) Graf Rostworowski, Diss. S. 81, Freiburg (Schweiz) 1911. 
noch nicht krystallisieren können. Es soll später näher untersucht werden. Dagegen gelang es leicht, auch die der Benzilsäure analoge I) iphenylenglykolsäure mit Thio-anisel zu paren.

9-(4'-Methylmercapto-phen!yl)-fluoren.

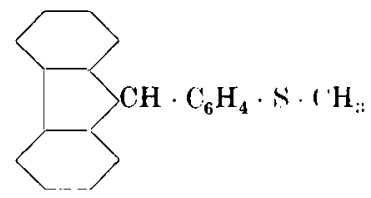

Wird die Kondensation der Diphenylenglykolsäure mit Phenylmethylthio-äther in der gleichen Weise durchgreführt, wie die mit Benzilsäure (s. o.), so färbt sich die benzolische Lösung bei halbstündigem Kochen braunschwarz; die entstandene Säure lässt sich der (zunächst mit verd. Salzsäure durchgeschüttelten) Lösung durch Ausschütteln mit warmer $\left(50^{\circ}\right)$, 5-proz. Soda entziehen. Beim C̈bersättigen der filtrierten alkalischen Lösung mit Essigsäure fällt die tertiäre Säure als schwach rusa gefärbte Masse aus. Wird sie aus kochendem Alkohol + Wasser krystallisiert, so geht sie unter Abspaltuny von Kohlendioxyd in das entsprechende Methanderivat über, das sich in langen Narleln ausscheidet. Diese ausserordentlich leichte Ibgabe von Kohlendioxyrl überrascht nicht, wenn man sich erinnert, lass die analog gebaute 9- (4'-Oxyphenyl)-fluoren-9-carbonsäure $\left.{ }^{1}\right)$,<smiles>CCCC(CC)(CCO)C(=O)O</smiles>

(aus I)iphenylenglykolsäure und Phenol) brim Kochen mit Sodalösung: oder Essigsäure-anhydrid gleichfalls Kohlendioxyrl ahspaltet.

Der vorliegende Methankörper schmilzt schon bei $126-128^{\circ}$, indem er sich fuchsinrot färbt. Er ist bei Siedetemperatur in Benzol oder Aceton leicht löslich, weniger in Eisessig, ziemlich schwer in Alkohol, während er von (hloroform schon in der Kälte leicht aufgenommen wird. Mit kalter konz. Schwefel-iilure iibergossen, geht er mit sehr schwach blauer Färbung in Lösung.

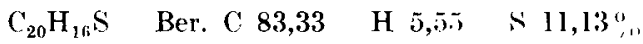

$$
\begin{aligned}
& \text { Gef. , 83,14 ,5,72 , 10,91",, }
\end{aligned}
$$

Auch in diesem Falle ist die angenommene stellung der Grupp . s. $\mathrm{CII}_{3}$ in $4^{\prime}$ nicht bewiesen.

1) Bistrzychi und. 2 . Weber, B. 43, 2498 (1910). 
9-(4'-Methylmercapto-phenyl)-fluoren-9-carbonsäure,

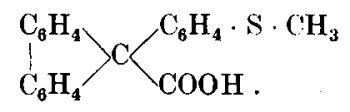

Um diese Carbonsäure in reinem Zustande ohne Krystallisation zu fassen, darf man sich nicht des Zinntetrachlorides, wie eben angegeben, bedienen, sondern muss folgendermassen verfahren: 2,3 gr Diphenylenglykolsäure und $1,4 \mathrm{gr}$ Phenylmethyl-thioäther werden in $15 \mathrm{~cm}^{3}$ Eisessig unter Erwärmen gelöst und zu dieser Lösung tropfenweise unter Kühlung und tüchtigem Lmschütteln $1,5 \mathrm{~cm}^{3}$ konz. Schwefelsäure gegeben, indem die Temperatur dauernd auf 40 bis höchstens $\mathbf{4 5 ^ { \circ }}$ gehalten wird.

Bald beginnt die entstandene Säure in grossen, fast farblosen Blättchen auszufallen. Nach fünf- bis sechs-stündigem Stehen bei Zimmertemperatur wird sie auf einem gehärteten Filter rasch durch Absaugen von der Mutterlauge befreit, mit warmem Wasser gewaschen und im Vakuum getrocknet. Sie ist dann rein.

$$
\begin{aligned}
& \mathrm{C}_{21} \mathrm{H}_{18} \mathrm{O}_{2} \mathrm{~S} \quad \text { Ber. C 75,90 H 4,82 }{ }^{\circ} \\
& \text { Gef. , 75,91 , } 5,07 \%
\end{aligned}
$$

Die Verbindung schmilzt unter starker Zersetzung bei etwa $170^{\circ}$. Dieser Punkt kann, je nach der Schnelligkeit des Erhitzens, um einige Grade schwanken. Die Säure ist in der Hitze leicht löslich in Aceton, ziemlich schwer in Chloroform, noch weniger in Ligroïn. Mit konz. Schwefelsäure auf $70^{\circ}$ erwärmt, geht sie mit indigblauer Farbe unter Kohlenoxydabspaltung in Lösung. Giesst man die erkaltete Lösung in Wasser, so fällt nichts aus, was auf die Bildung von Sulfosäuren hinweist. Die tertiäre Säure aus Diphenylenglykolsäure und Anisol') lieferte unter den gleichen Bedingungen ebenfalls kein Carbinol.

Die letztbeschriebene Arbeitsweise (Anwendung von EisessigSchwefelsäure bei $40-45^{\circ}$ ) gestattet auch die Vereinigung von Benzilsäure mit freiem Thiophenol. Über das Produkt dieser Reaktion, dessen Konstitution wir noch nicht mit Bestimmtheit angeben können, wèrden wir später berichten.

Freiburg i. Uechtland, I. Chem. Laboratorium der Iniversität.

1) Bistrzychi und v. Weber, B. 43, 2504 (1910). 